\title{
Estratégia Saúde da Família e internações por condições sensíveis à Atenção Básica: caminhos possíveis
}

\author{
Family Health Strategy and hospitalization for conditions sensitive to Primary Care: possible ways
}

Estrategia de Salud Familiar y hospitalizaciones sensibles a la Atención Básica: posibles formas

Lívia dos Santos Sanches Carriello ${ }^{1}$, Ândrea Cardoso de Souza ${ }^{2}$

Como citar esse artigo. Carriello, LSS;

Souza, AC. Estratégia Saúde da Família e internações por condições sensíveis à Atenção Básica: caminhos possíveis. Revista Pró-UniverSUS. 2019 Jul./Dez.; 10 (2): 97-100.

\begin{abstract}
Resumo
Introdução: O objeto desse estudo é a relação da Estratégia Saúde da Família com as internações por condições sensíveis à atenção básica cujo objetivo é avaliar de que forma esse nível de atenção à saúde interfere nas internações de alcance da Atenção Básica referentes à hipertensão arterial e diabetes mellitus. Materiais e Métodos: trata-se de pesquisa qualitativa onde realizou-se entrevistas semiestruturadas com as equipes de Saúde da Família de um município do Estado do Rio de Janeiro. Os dados foram analisados tendo como referência o processo de trabalho e a micropolítica do cuidado e da gestão. $\mathrm{O}$ estudo foi aprovado pelo Comitê de Ética em Pesquisa do Hospital Universitário Antônio Pedro da Universidade Federal Fluminense. Resultados esperados: pretende-se desenvolver fluxograma para acompanhar estas internações e, então, elaborar oficina de Educação Permanente em Saúde voltada para discussão da atenção à hipertensão arterial e ao diabetes mellitus. Através de fluxogramas é possível otimizar a comunicação entre as redes de atenção à saúde e a gestão permitindo melhor acesso e redução nas internações por hipertensão e diabetes.
\end{abstract}

Palavras-chave: Atenção Primária à Saúde, Avaliação em Saúde, Estratégia Saúde da Família, Hospitalização.

\begin{abstract}
Objectives: Introduction: The object of this study is the relationship between the Family Health Strategy and hospitalizations due to conditions sensitive to primary care with objective to evaluate how this level of health care interferes with primary care admissions for hypertension and diabetes mellitus. Materials and Methods: this is a qualitative research where semi-structured interviews were conducted with the Family Health teams of a city in the state of Rio de Janeiro. The data were analyzed by reference to the work process and the micro-policy of care and management. The study was approved by the Research Ethics Committee of the Hospital UniversitárioAntônio Pedro of the Universidade Federal Fluminense. Expected Results: It is intended to develop flowchart to accompany these admissions and, then, elaborate workshop of Permanent Health Education focused on discussion of attention to hypertension and diabetes mellitus. Through flow charts it is possible to optimize the communication between health care networks and management allowing better access and reduction in hospitalizations due to hypertension and diabetes.
\end{abstract}

Keywords: Primary Health Care, Health Evaluation, Family Health Strategy, Hospitalization.

\section{Resumen}

Objetivos: Introducción: el objeto de este estudio es la relación de la estrategia salud de la familia con las internaciones por condiciones sensibles a la atención básica com lo objetivo de evaluar cómo interfiere este nivel de atención a la salud en las internaciones de alcance de la atención básica referentes a la hipertensión arterial y diabetes mellitus. Materiales y métodos: se trata de investigación cualitativa donde se realizaron entrevistas semiestructuradas con los equipos de salud de la familia de un municipio del estado de río de janeiro. los datos fueron analizados teniendo como referencia el proceso de trabajo y la micropolítica del cuidado y de la gestión. El estudio fue aprobado por el comité de ética en investigación del hospital universitario antonio pedro de la universidad federal fluminense. Resultados esperados: se pretende desarrollar fluxograma para acompañar estas internaciones y luego elaborar taller de educación permanente em salud enfocado para discusión de la atención a la hipertensión arterial y a la diabetes mellitus. Através de fluxogramas es posible optimizar la comunicación entre las redes de atención a la salud y la gestión permitiendo un mejor acceso y reducción en las internaciones por hipertensión y diabetes.

Palabras clave: Atención Primaria de Salud; Evaluación en Salud; Estrategia Salud de la Familia; Hospitalización.

Afiliação dos autores: 1. Enfermeira. Mestranda do programa de Mestrado Profissional em Ensino na Saúde/MPES, UFF, RJ, Brasil. Email: liviacarriello@gmail.com ORCID: https://orcid.org/0000-0001-6 416-1995

2. Enfermeira. Professora do Programa de Mestrado Profissional em Ensino na Saúde/MPES, UFF, RJ, Brasil. Email: andriacsouza@gmail.com ORCID: https://orcid.org/0000-0002 $-6549-8634$ 


\section{Introdução}

A Estratégia Saúde da Família (ESF) vem se moldando no interior das políticas de saúde, com intuito de promover, expandir e consolidar a Atenção Primária no Brasil. Ao longo de sua consolidação e de acordo com as realidades e necessidades locais, a ESF agregou novas dimensões à sua dinâmica, ampliando seu alcance e considerando o modo de vida da população. Em consequência, vem exigindo no processo de trabalho das suas profissionais novas qualificações e atributos.

Ao se abordar o processo de trabalho em saúde e a qualidade do serviço ofertado, utilizam-se alguns indicadores que contribuem para melhor avaliação das práticas realizadas. Todo instrumento capaz de avaliar a qualidade da Atenção Básica, seu objeto de trabalho e objetivos que ela propõe alcançar como porta de entrada do sistema, auxilia no fortalecimento desse nível de atenção. A realização periódica do processo avaliativo é uma importante estratégia para o fortalecimento da Atenção Básica, onde a equipe, ativadora desse movimento, percebe a sua prática, tem uma discussão crítica sobre a organização dos serviços e descobre seu potencial de mudança na vida do usuário ${ }^{1}$.

Nesse aspecto, um dos indicadores de avaliação e monitoramento da efetividade da ESF é representado pelas taxas de internações por condições sensíveis à Atenção Básica (ICSAB). Essas condições são problemas de saúde que deveriam ter sua resolutividade nesse primeiro nível de atenção, quando diagnosticadas de forma oportuna e precoce. As ICSAB agrupam diferentes causas de internação por problemas de saúde tipicamente gerenciados no primeiro nível de atenção à saúde, ou seja, aqueles para as quais a necessidade de hospitalização poderia ter sido evitada com atendimento ambulatorial. Este indicador surgiu nos Estados Unidos ao final dos anos 80 para identificar a população sem acesso à atenção ambulatorial e estudar o impacto financeiro sobre o sistema de saúde ${ }^{2}$.

Altas taxas de internações de uma população por condições sensíveis à atenção básica podem indicar sérios problemas do desempenho desse nível de atenção e estão associadas à baixa cobertura dos serviços, à baixa resolutividade e ineficácia ${ }^{3}$.

No Brasil, a publicação da portaria do Ministério da Saúde MS/GM no 221, de 17 de abril de 2008, apresentou a Lista Brasileira de Internações por Condições Sensíveis à Atenção Primária. Entre os problemas de saúde elencados destacam-se as doenças preveníveis por imunização, gastroenterites infecciosas, anemia, deficiências nutricionais, infecções de ouvido, narize garganta, pneumonias bacterianas, asma, doenças pulmonares, hipertensão, angina, diabetes mellitus, infecção no rim e trato urinário ${ }^{4}$.

Destacam-se nessa lista as doenças crônicas não transmissíveis (DCNT) entre elas, a hipertensão arterial e o diabetes mellitus,que representam uma expressiva e crescente demanda em termos de atenção em saúde exigindo novasabordagens para a garantia de acompanhamento e continuidade do cuidado e de monitoramento. O Brasil vive uma transição demográfica acelerada, ou seja, a população encontrase em rápido processo de envelhecimento significando maior ocorrência das condições crônicas visto que elas acometem os segmentos de maior idade 5 .

O diagnóstico de uma doença crônica, a adesão ao tratamento, as condições socioeconômicas, culturais e emocionais que interferem nesse processo, devem ser estudados para melhor compreender as fragilidades no acompanhamento da mesma. Daí a importância de investimentos na atenção básica para o manejo adequado dessas doenças e consequente redução das internações.

Partindo dessa realidade e do atrelamento entre processo de trabalhoxICSABxlevantam-se as seguintes questões: Como o processo de trabalho pode favorecer a ocorrência de ICSAB? De que forma os profissionais de saúde percebem seu trabalho na vida do usuário hipertenso e diabético? Qual ferramenta de gestão poderia ser implementada para auxiliar na redução das ICSAB?

Dessa forma, o objeto desse estudo é a relação das internações por condições sensíveis a atenção básica com a Estratégia Saúde da Família.

Sendo assim, o objetivo desse projeto é analisar de que forma o processo de trabalho da Estratégia Saúde da Família interfere nas internações por condições sensíveis à atenção básica referentes à hipertensão arterial e ao diabetes mellitus.

É crucial avaliar de que forma o processo de trabalho da ESF vem interferindo nas ICSAB, principalmente no tocante à hipertensão arterial e ao diabetes mellitus, que são problemas de saúde de curso longo e que exigem permanente cuidado. Também é importante discutir alternativas possíveis para a reversão da realidade abordada.

Estudos que avaliem os coeficientes de ICSAB, suas tendências e sua relação com a Estratégia Saúde da Família são importantes para o planejamento e a formulação de políticas e estratégias que impactem na redução destes coeficientes ${ }^{6,7}$.

\section{Metodologia}

Trata-sede nota prévia de pesquisa qualitativa do tipo pesquisa-ação. A pesquisa-ação tem como proposta uma ação deliberada de transformação da realidade investigada e a produção do conhecimento. Pretende agregar na formação do sujeito participativo e autônomo, inserindo-o nos resultados apreendidos com a pesquisa durante do processo investigativo ${ }^{8}$. 
Este estudo foi conduzido a partir da técnica de entrevista semiestruturada para coleta de dados. Por meio das entrevistas o pesquisador busca apreender as falas dos participantes do estudo. Utilizou-se duas modalidades de perguntas: abertas, onde o entrevistado aborda de forma livre $\mathrm{o}$ assunto questionado, e fechadas, com perguntas previamente estabelecidas ${ }^{9}$.

Os dados foram coletados após submissão e aprovação do Comitê de Ética em Pesquisa do Hospital Universitário Antônio Pedro da Universidade Federal Fluminense, com parecerde número 3.165.518, respeitando-se a Resoluções do Conselho Nacional de Saúde no 466/2012 e no 510/2016.

Os participantes da pesquisa entrevistados foram os componentes da equipe mínima da ESF que deve ser composta por, no mínimo, médico, enfermeiro, auxiliar ou técnico de enfermagem e agentes comunitários de saúde ${ }^{10}$.

Foram entrevistados no total de 24 componentes das oito equipes que estão diretamente associados ao acompanhamento dos usuários hipertensos $\mathrm{e}$ diabéticos.

Para que fosse possível uma representatividade igualitária do número de participantes por categoria profissional e assim, ter um conjunto de ideias e pensamentos das diferentes profissões, optou-se por definir que em cada unidade de saúde seriam entrevistados três profissionais previamente definidos com o intuito de contemplar as perspectivas de cada um. Para a seleção desses profissionais foi realizado um sorteio para que não houvesse parcialidade na escolha.

A aplicação dos questionários foi realizada individualmente em ambiente reservado e após leitura e assinatura do Termo de Consentimento Livre e Esclarecido (TCLE). As entrevistas foram gravadas e transcritas com o objetivo de aproveitamento amplo das falas e dos sentidos nelas contidas. Os participantes da pesquisa terão seus nomes preservados sendo substituídos por nomenclatura fictícia.

Após a coleta de dados e transcrição das entrevistas, as informações serão analisadas e categorizadas. Para fins deste estudo, as informações serão analisadas à luz do referencial teórico adotado tendo como conceitos-guia o processo de trabalho e a micropolítica do cuidado e da gestão. Os dados serão agrupados em conformidade com as temáticas viabilizando uma análise mais aprofundada. Para isso, será utilizada o método da Análise de Conteúdo que representa um conjunto de técnicas de pesquisa que tem por objetivo a busca de significados de um documento. Ela se volta para a compreensão tanto dos conteúdos quanto das evidências tendo como ponto de partida a comunicação ${ }^{11}$.

Este estudo se dará especificamente no município de Cantagalo. Localizado na Região Serrana do Estado do Rio de Janeiro, a 200 km de distância da capital,
Cantagalo possui uma área de $746,928 \mathrm{~km}^{2} \mathrm{e}$ densidade demográfica de 26,47 habitante por $\mathrm{km}^{2}$. Segundo o censo de 2010, a população é de 19.830 habitantes sendo 9.831 homens e 9.999 mulheres $^{12}$.

A cobertura populacional da Estratégia Saúde da Família é de 100\%, com uma equipe de Saúde Bucal modalidade I para cada ESF. O município aderiu ao $3^{\circ}$ Ciclo do Programa de Melhoria do Acesso e da Qualidade (PMAQ) com as 8 equipes de ESF e ESFB modalidade I (idem). Serão avaliadas as oito equipes de Saúde da Família, sendo quatro localizadas na sede municipal e quatro localizadas em seus respectivos distritos.

\section{Resultados Esperados}

Este estudo tem como proposta um produto que possa dar resposta a sua problemática central. Pretende-se produzir e implementar um fluxograma que aponte com mais clareza o percurso de entrada e saída do usuário no sistema de saúde pertinente à assistência à hipertensão arterial e ao diabetes mellitus, ou seja, almeja-se um fluxograma de informações que possa avaliar e monitorar as internações por condições sensíveis à atenção básica no município de Cantagalo sinalizando as suas causas e apontando problemas no acesso ao cuidado nas unidades de Saúde de Família.

O fluxograma é uma ferramenta gerencial que descreve o trajeto do atendimento do usuário a partir de sua entrada no serviço de saúde. Ele permite um olhar sobre o percurso da produção da assistência em saúde possibilitando a detecção de problemas durante esse fluxo. Possibilita a detecção de "áreas de sombra" até então não percebidas que vai na contramão de uma oferta de cuidado de qualidade onde o usuário tem o papel central ${ }^{13}$.

Espera-se com o fluxograma, otimizar a comunicação entre hospital-unidade de saúde-gestão e a partir deste, subsidiar a elaboração de oficinas de Educação Permanente em Saúde (EPS) que possibilite discussões sobre a interfacedo processo de trabalho na Estratégia Saúde da Família e as ICSAB.

A EPS possibilita a aprendizagem no trabalho e para o trabalho. Por meio dela pretende-se sensibilizar as equipes de Saúde da Família de Cantagalo acerca da importância do processo de trabalhono cuidado aos usuários hipertensos e diabéticos,contribuindo assim para a melhoria dos indicadores relacionados não somente à hipertensão arterial e ao diabetes mellitus como também a outras doenças da lista de condições sensíveis a Atenção Básica.

\section{Referências}

1. Castanheira ERLet al. Desafios para avaliação na atenção básica no Brasil: a diversidade de instrumentos contribui para a instituição de uma cultura avaliativa? In: AKERMAN, Marco; FURTADO, Juarez Pereira (org). Práticas 
de avaliação em saúde no Brasil - diálogos. Porto Alegre: Rede Unida, 2015. 374p. Disponível em: <http://historico.redeunida.org.br/editora/bibliotecadigital/serie-atencao-basica-e-educacao-na-saude/praticas-de-avaliacao-emsaude-no-brasil-dialogos-pdf $>$ Acesso em 20 setembro2018.

2. NedelFB et al .Conceptual and methodological aspects in the study of hospitalizations for ambulatory care sensitive conditions.Ciênc. saúdecoletiva, Rio de Janeiro ,2011; 16, supl. 1:1145-1154. Disponível em: $<$ http://www.scielo.br/scielo.php?script $=$ sci arttext\&pid=S1413812320110 00700046\&lng=en\&nrm=iso $>$. Acesso em 26 Maio 2018.

3. Alfradique MEet al. Internações por condições sensíveis à atenção primária: a construção da lista brasileira como ferramenta para medir o desempenho do sistema de saúde (Projeto ICSAP - Brasil). Cad. Saúde Pública, Rio de Janeiro , 2009June.; 25(6): 1337-1349. Disponível em $<$ http://www.scielo.br/scielo.php?script $=$ sci_arttext\&pid=S0102311X20090 00600016\&lng=en\&nrm=iso $>$. Acesso em 20 Maio 2018.

4. Brasil, Ministério da Saúde. Portaria no 221, de 17 de abril de 2008. Publica a lista brasileira de internações por condições sensíveis à atenção primária. Diário Oficial da União, Brasília, p. 70, 18 abr. 2008. Seção 1. Disponível em $:<$ http://bvsms.saude.gov.br/bvs/saudelegis/sas/2008/prt0221 17042008. html $>$ Acesso em 22 maio 2018

5. Mendes EV. O cuidado das condições crônicas na Atenção Primária à Saúde: o imperativo da consolidação da Estratégia da Saúde da Família. Brasília: Organização Pan-americana da Saúde, 2012. 512p. Disponível em $<$ http://bvsms.saude.gov.br/bvs/publicacoes/cuidado_condicoes_atencao_ primaria_saude.pdf $>$ Acesso em 28 agosto 2018

6. Morimoto T, CostaJSD da. Internações por condições sensíveis à atenção primária, gastos com saúde e Estratégia Saúde da Família: uma análise de tendência. Ciênc. saúde coletiva, Rio de Janeiro , v. 22, n. 3, p. 891-900, Mar. 2017 . Disponível em <http://www.scielo.br/scielo.php?script=sci_ arttext\&pid $=$ S1413-81232017002300891\&lng=en\&nrm $=$ iso $>$. Acesso em 22 Maio 2018.

7. Arruda JS de; Costa JSD da. Internações por condições sensíveis à atenção primária em Novo Hamburgo, Rio Grande do Sul. Revista Brasileira de Medicina de Família e Comunidade, [S.1.], 2017 maio;12(39):1-11, ISSN 2179-7994. Disponível em: <https://rbmfc.org.br/rbmfc/article/ view/1256/840>. Acesso em: 09 out 2018.

8. Tanajura LLC, Bezerra AAC. Pesquisa-Ação sob a ótica de René Barbier e MichellThiollent: aproximações e especificidades metodológicas. Rev. Eletrônica Pesquiseduca, Santos, 2015jan.-jun.; 07(13):10-23. Disponível em $\quad<$ :http://periodicos.unisantos.br/index.php/pesquiseduca/article/ download/\%20408/pdf> Acesso em 10 outubro 2018

9. Minayo, MC de S. (org),Deslandes SF, Gomes R. Pesquisa social - teoria, método, criatividade. Petrópolis: Editora Vozes, 2011. 112p.

10. Brasil. Ministério da Saúde. Secretaria de Atenção à Saúde. Departamento de Atenção Básica. Estratégias para o cuidado da pessoa com doença crônica: hipertensão arterial sistêmica / Ministério da Saúde, Secretaria de Atenção à Saúde, Departamento de Atenção Básica. - Brasília: Ministério da Saúde, 2013. Disponível em $:<$ http://bvsms.saude.gov.br/bvs/publicacoes/ estrategias_cuidado_pessoa_doenca_cronica.pdf> Acesso em 12 out 2018.

11. Campos CJG. Método de análise de conteúdo: ferramenta para a análise de dados qualitativos no campo da saúde. Rev. bras. enferm. Brasília, 2004out.;57(5):611-614. . Disponível em <http://www.scielo.br/scielo. php?script $=$ sci arttext\&pid $=\mathrm{S} 003471672004000500019 \& \operatorname{lng}=\mathrm{pt} \& \mathrm{nrm}=\mathrm{is}$ o>. Acesso em 26 nov 2018.

12. IBGE, Instituto Brasileiro de Geografia e Estatistica. Cantagalo População. 2018. Disponível em <https://cidades.ibge.gov.br/brasil/rj/ cantagalo/panorama> Acesso em 25 maio 2018.

13. Franco, TB. O Uso do Fluxograma Descritor e Projetos Terapêuticos para Análise de Serviços de Saúde, em apoio ao Planejamento: O caso de Luz - MG. IN: O TRABLHO EM SAÚDE: Olhando e experienciando o SUS no cotidiano"; Merhy, E.E.; Franco, T.B. et al; HUCITEC, São Paulo, 2003. Disponível em https://www.pucsp.br/prosaude/downloads/territorio/ fluxograma_descritor_e_Projetos_terapeuticos_-_Prof_Dr_Tulio_Franco. pdf 